\title{
A Divide-and-conquer Strategy in Shape from Shading Problem
}

\author{
Seong Ik CHO, Hideo SAITO, and Shinji OZAWA \\ Ozawa-Saito Lab, \\ Department of Electrical Engineering, \\ Faculty of Science and Technology, \\ Keio University, Yokohama-shi, 223, Japan \\ E-mail : \{sicho@, saito@ and ozawa@\}ozawa.elec.keio.ac.jp
}

\begin{abstract}
A divide-and-conquer strategy in shape from shading problem under fully perspective condition is proposed for the information recovery of book surfaces. The whole recovery process is composed of three sequential steps : preprocessing, apparent shape recovery, and ortho-image generation. Pure shade images are extracted at preprocessing step by introducing phenomenological model of interreflection and by removing pigment parts from observed images. Using existing invariances, equations of slope and that of ortho-image being explicit are derived from equation of shading and that of observation being implicit. Recurrence relation is derived from definition of mean slope in discrete image. Theoretically, it become possible to recover unique shape without iteration using derived equations in case of Lambertian cylinder. However, a feed-back shape recovery process is implemented as practical algorithm in order to overcome self-shadows. Results of simulations and real experiments show the properness and acceptability of the proposed strategy and implemented algorithms.
\end{abstract}

\section{Introduction}

In this paper, a divide-and-conquer strategy is discussed in solving shape from shading (SFS) problem of book surface under fully perspective condition.

The observing environments are composed of two light sources and one camera which are the usual setting in industrial applications. For surface of book, it is assumed that albedo of paper part is homogeneous, that global variations of depth exist along one direction only, and that surface of book has matte reflectance characteristics following Lambertian reflectance[2].

There are two major difficulties in this problem of

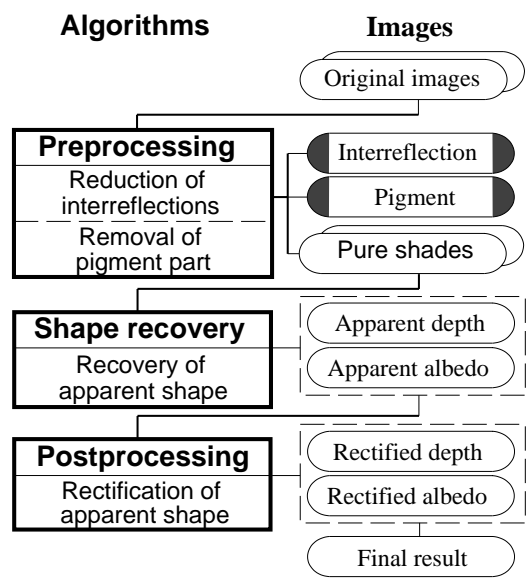

Figure 1. Flow diagram of the whole process proposed for shape recovery of book surface.

real situation. One comes from obstacles like nonconstant albedos, self-shadows, interreflections[7][9] and discontinuity of shading in folded part. Another comes from perspective effect which generally increases the unknowns and which makes the problem implicit because prior information of depth, that is the solution itself, is required to solve the problem.

Parts of the idea proposed in this paper are connected with several previous researches on perspective light condition[1][5][6], on recovery of book surface[9][10], on perspective observation[4][3][6], and on using photometric ratio[11].

The purpose of this paper is to propose a fast way of shape recovery using shading information.

For this, a divide-and-conquer strategy, which divides complicated SFS problem into sequential and simple ones, is proposed as the fundamental idea. By this strategy, the whole recovery process become large- 


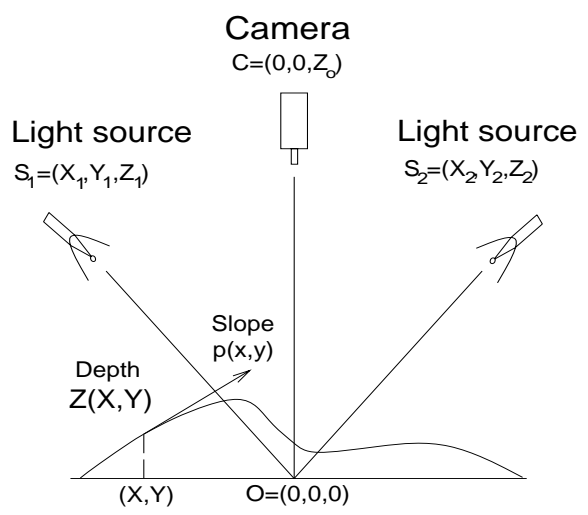

(a) Position of light sources, camera, and object surface defined in world coordinate.

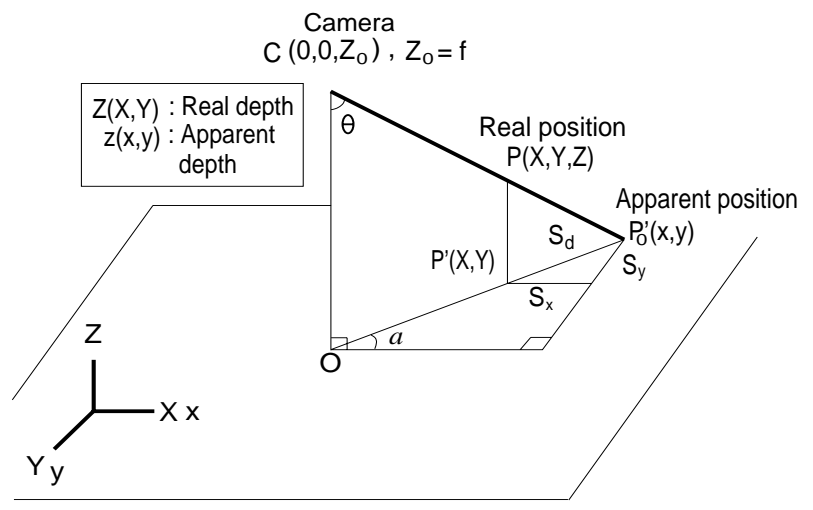

(b) Apparent position shift defined by the relation of two coordinate systems.

Figure 2. World and pixel coordinates

ly divided into three steps of processing as is shown in Fig. 1. Preprocessing is designed for extracting pure shade images from observed images of book surface. Shape information is recovered through two sequential steps because this strategy divides recovery problem into two in order to solve fully perspective SFS problem. This strategy makes it possible to recover shape of Lambertian cylinder uniquely without iteration when there is no self-shadow and no discontinuity of shading.

This paper is arranged as follows. Mathematical problem and theoretical solution will be discussed in Sect. 2 and Sect. 3, respectively. Implementation of algorithms will be discussed in Sect. 4 , and results of simulations and experiments will be shown in Sect.5. Finally, discussion will be done in Sect. 6 .

\section{Problem formulation}

Under fully perspective condition, two equations based on world and pixel coordinates should be considered simultaneously in order to describe observed reflectance of each pixel on image. Equation of shad- ing based on world coordinate is used for describing amount of perspective shading, whereas equation of observation defined by the relation of both coordinates is used for describing deformations of object under perspective observation.

For discriminating two coordinates, $(X, Y, Z)$ and $(x, y, z)$ will be used for world and pixel coordinates respectively throughout this paper. We will denote location of $i$-th light source as $\left(X_{i}, Y_{i}, Z_{i}\right)$, height of camera as $Z_{o}$, local slope along $X$ axis as $p$, and product of departed strength of light and albedo of surface as $I_{0} \rho$, respectively. The location of each term in world coordinate, and the concept of apparent position is shown in Fig. 2.

\subsection{Equation of shading}

Amount of shading $L_{i}$ by perspectively incident light source in Lambertian model[2] becomes

$$
\begin{gathered}
L_{i}=I_{0} \rho \frac{\cos \theta_{i n c}}{D_{i}^{2}}=I_{0} \rho \frac{-\Delta_{X i} p+\Delta_{Z i}}{D_{i}^{3} \sqrt{1+p^{2}}}, \\
p \equiv \frac{\partial Z}{\partial X}, \quad \frac{\partial Z}{\partial Y} \equiv 0, \quad D_{i} \equiv \sqrt{\Delta_{X i}^{2}+\Delta_{Y i}^{2}+\Delta_{Z i}^{2}}, \\
\Delta_{X i} \equiv X_{i}-X, \quad \Delta_{Y i} \equiv Y_{i}-Y, \quad \Delta_{Z i} \equiv Z_{i}-Z .
\end{gathered}
$$

\subsection{Equation of observation}

Equation of perspective observation found in usual text books is described by two variables : focal length and height of camera. Without loosing generality, it is possible to extend focal length equivalent with $Z_{o}$ as is shown in Fig. 2 (b) in order to describe relation of both coordinates simultaneously on image plane.

In Fig. 2 (b), a point $P(X, Y, Z)$ on object surface is recorded at $P_{o}^{\prime}(x, y)$ by apparent shift $S_{d}$. Since $S_{d} \equiv$ $Z \tan \theta$ and $\overline{O P_{o}^{\prime}} \equiv Z_{o} \tan \theta, S_{x}$ becomes

$$
S_{x}=S_{d} \cos \alpha=S_{d} \frac{x}{\overline{O P_{o}^{\prime}}}=x \frac{Z}{Z_{o}},
$$

and $S_{y}$ become $y \frac{Z}{Z_{o}}$ in the same way.

Since $X=x-S_{x}$ and $Y=y-S_{y}$ in the figure, following equation of observation is derived

$$
X=x\left(1-\frac{Z}{Z_{o}}\right), \quad Y=y\left(1-\frac{Z}{Z_{o}}\right), \quad Z \equiv Z(X, Y) \text {. }
$$

\subsection{Recovery problem}

Shape from shading in fully perspective condition is a way of recovering shape of surface using observed shade images and using the equations (1) and (3).

At a glance, both equations look like containing five unknowns $\left(X, Y, Z, I_{0} \rho, p\right)$ because of undetermined depth $Z(X, Y)$. However, $(X, Y)$ in eq. (3) can be determined when prior information of depth $Z(X, Y)$ is available. Moreover, local slope is directly connected 
with depth. Therefore, it can be intuitively acknowledged that actual unknowns of this problem are not five but only two $\left(Z, I_{0} \rho\right)$, and that difficulty is in implicitness of problem because $Z(X, Y)$ should be known priorily in order to minimize unknowns.

Therefore, the key of problem treated in this paper is on how to get prior value of depth because reduction of unknowns and resolving implicitness shall be done naturally from prior information of depth.

\section{Equations used in shape recovery}

Using existing invariances, we will derive three new equations : equation of slope and recurrence relation which makes it possible to recover apparent shape defined in pixel coordinate, and equation of ortho-image which will be used in generating ortho-image of shape and albedo.

\subsection{Invariant characteristics}

Shape should be recovered at apparent position $P_{o}^{\prime}(x, y)$ because shade images are source of shape information. Angle between surface normal and incident light ray is invariant even in perspective observation because these are defined in world coordinate. Amount of shading is invariant as well by Lambertian assumption, that is $L(x, y) \equiv L(X, Y)$, when distance variation between camera and surface can be ignored. Consequently, recovered slope $p(x, y)$ becomes equivalent with $p(X, Y)$, and as a result, $z(x, y) \equiv Z(X, Y)$ even in perspective observation.

\subsection{Transformed shading equation}

By virtue of invariance of depth, it is possible to substitute eq. (3) into (1). By this substitution, transformed shading equation is derived

$$
\begin{gathered}
L_{i}=I_{0} \rho \frac{-\delta_{x i} p+\delta_{z i}}{d_{i}^{3} \sqrt{1+p^{2}}}, \\
d_{i} \equiv \sqrt{\delta_{x i}^{2}+\delta_{y i}^{2}+\delta_{z i}^{2}}, \quad \delta_{x i} \equiv X_{i}-x\left(1-\frac{z}{Z_{o}}\right), \\
\delta_{y i} \equiv Y_{i}-y\left(1-\frac{z}{Z_{o}}\right), \quad \delta_{z i} \equiv Z_{i}-z, \quad z \equiv z(x, y) .
\end{gathered}
$$

This substitution eliminates two unknowns $(X, Y)$, and replaces depth $Z(X, Y)$ into $z(x, y)$, maintaining generality of problem. As a natural consequence, original problem becomes a problem of apparent shape recovery because eq. (4) is defined in pixel coordinate.

\subsection{Equation of slope}

The equation of slope can be derived by calculating photometric ratio[11] of two transformed shading equations,

$$
\frac{L_{1}}{L_{2}}=\frac{\left(-\delta_{x 1} p-\delta_{z 1}\right) d_{2}^{3}}{\left(-\delta_{x 2} p-\delta_{z 2}\right) d_{1}^{3}},
$$

in order to eliminate unknown $I_{0} \rho$. By solving this equation with respect to slope $p$ on the assumption of known $z(x, y)$, equations of slope is derived

$$
p^{D}=\frac{L_{1} \delta_{z 2} d_{1}^{3}-L_{2} \delta_{z 1} d_{2}^{3}}{L_{1} \delta_{x 2} d_{1}^{3}-L_{2} \delta_{x 1} d_{2}^{3}} .
$$

When single image is available, this becomes

$$
\begin{gathered}
p_{i}^{S}=\frac{B \pm \sqrt{B^{2}-A C}}{A}, \quad \text { for } I_{0} \rho=1 . \\
A \equiv \delta_{x i}^{2}-L_{i}^{2} d_{i}^{6}, \quad B \equiv \delta_{x i} \delta_{z i}, \quad C \equiv \delta_{z i}^{2}-L_{i}^{2} d_{i}^{6} .
\end{gathered}
$$

Equation (7) is useful when image contains selfshadow. The \pm sign appeared in this equation can be discriminated easily by comparing the slopes $p^{D}$ and $p_{i}^{S}$ on shadowless zone.

\subsection{Recurrence relation}

Since equation of slope is derived by assuming known depth, depth should be determined prior to slope. Recurrence relation makes it possible to determine depth priorily.

In discrete image, since mean slope is defined as

$$
p(x-1, y)=\frac{z(x, y)-z(x-2, y)}{2 \epsilon}, \quad \epsilon \approx 1,
$$

following recurrence relation can be derived with natural boundary condition

$$
\begin{gathered}
z(x, y)=z(x-2, y)+2 p(x-1, y), \\
z(0, x) \equiv 0, \quad z(1, y) \equiv p(0, y) .
\end{gathered}
$$

\subsection{Equation of ortho-image}

Since shape is recovered in pixel coordinate, this shape should be corrected into ortho-image of shape by applying equation of observation.

Equation of ortho-image is derived by inverting eq. (3) for easy interpolation. This becomes

$$
x=[X] \frac{Z_{o}}{Z_{o}-z}, \quad y=[Y] \frac{Z_{o}}{Z_{o}-z},
$$

where $([X],[Y])$ denotes position of each pixel in orthoimage expressed by integer value. This equation should be applied for both the albedo and shape images.

\subsection{Theoretical recovery process}

Shape recovery starts from boundary condition of eq. (10) which gives initial depth. Slope can be determined by applying eq. (6) using prior information of depth and using observed shade images. Recurrence relation of eq. (9) gives depth information of next pixel using calculated slope. Then, slope at next pixel can be determined by applying eq. (6) in the same way.

By this way of propagation, in ideal case, shape information can be recovered uniquely without iteration. 


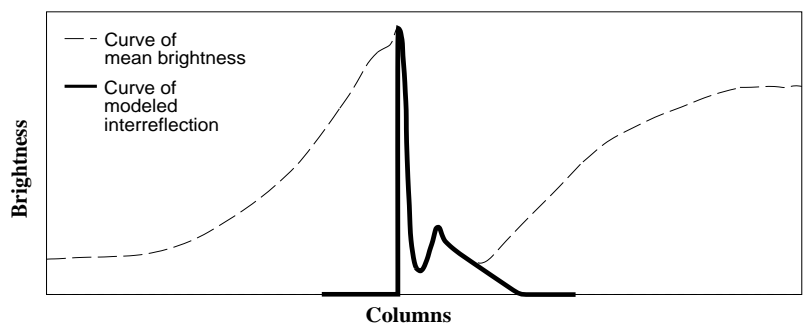

Figure 3. Concept of phenomenological model of interreflection.

\subsection{Compatibility and uniqueness}

Equation (4) becomes equivalent with eq. (1) when distance to camera becomes infinity because $(x, y)$ becomes as same as $(X, Y)$. Therefore, the proposed recovery process is compatible with orthographic condition.

In ideal case, uniqueness of recovered shape is guaranteed naturally because recovery can be done mechanically without any ad hoc constraint.

\section{Implementation of algorithms}

Shape recovery problem in real situation is not simple because there are usually non-constant albedos, self-shadows, interreflections, and other unknown factors within observed shade images of book surface.

Within implemented algorithms, column-wise mean information of shade images will be used for reducing errors caused by local noises. However, this will reduce accuracy of recovered informations as well.

Pure shade images have been extracted from observed shade images by introducing ad hoc approach. Recovery of shape has been done using pure shade images by introducing a feed-back process which overcomes self-shadows.

\subsection{Preprocessing}

Effects of interreflections have been reduced from observed shade images by introducing phenomenological model, and hence pure shade images have been extracted by removing pigment parts.

\subsubsection{Reduction of interreflections}

Interreflections, which are occurred mainly on concave parts[8][7][9] of book, are hard to remove because of many unknown factors. Phenomenological model determined from mean reflectance curve of observed shade image is proposed for reducing effect of interreflections. Figure 3 shows the concept of this model. Dotted curve corresponds to column-wise mean reflectance of shade image. It can be easily acknowledged

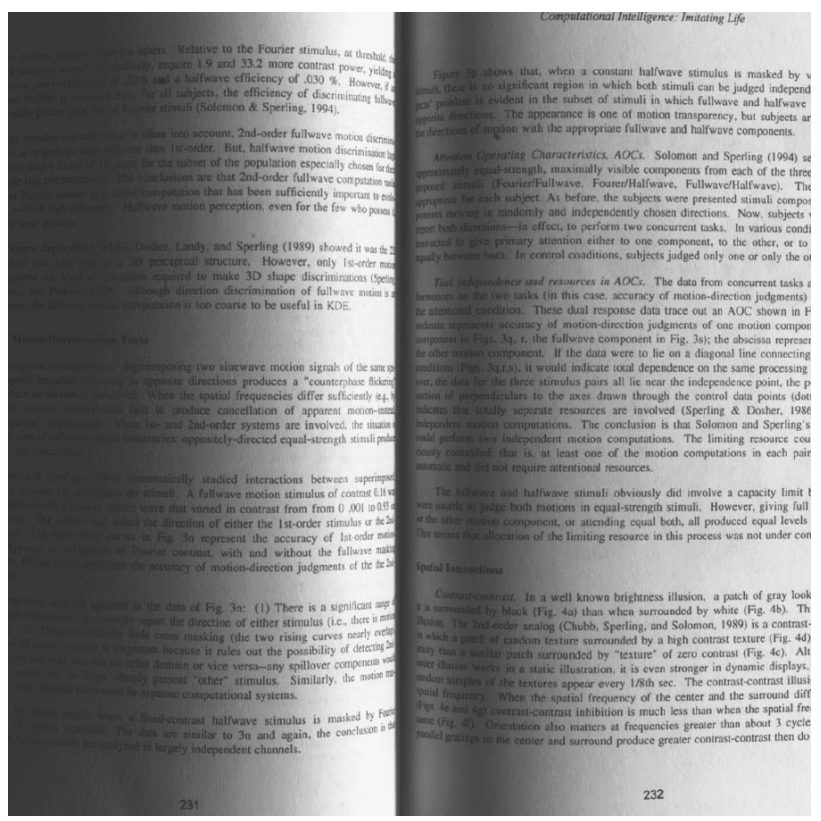

Figure 4. One of original shade image incident from right light source.

that reflectance peak of folded part (bold line) has been caused by effect of interreflections.

Phenomenological model $L_{i}^{G}(x)$ is obtained by following mean reflectance curve in folded part and by extrapolating. Local effect can be estimated using this model, and can be removed from shade image. Local effect $L_{i}^{L}(x, y)$ is defined as

$$
L_{i}^{L}(x, y) \equiv a(x, y) L_{i}^{G}(x)+b(x, y) .
$$

Parameters $a$ and $b$ are calculated by minimizing fitting error of both sides of eq. (12).

\subsubsection{Extraction of pure shade images}

Since local variations of reflectance are caused by the existence of pigments (character parts), pigment parts can be removed using the local statistics of image. The amounts of local reflectance variation are expressed as

$$
V(x, y)=\frac{\sigma_{I}(x, y)}{\bar{I}(x)},
$$

where $\sigma_{I}$ denotes local standard deviation and $\bar{I}$ denotes column-wise mean reflectance. The denominator is used for compensating global reflectance variation caused by shading. Paper part can be extracted through histogram analysis of variation image. The pigment pixels are substituted by new values interpolated from the nearest paper pixels.

Figure 4 shows one of observed images, and Fig. 5 shows the extracted pure shade image. Although this 

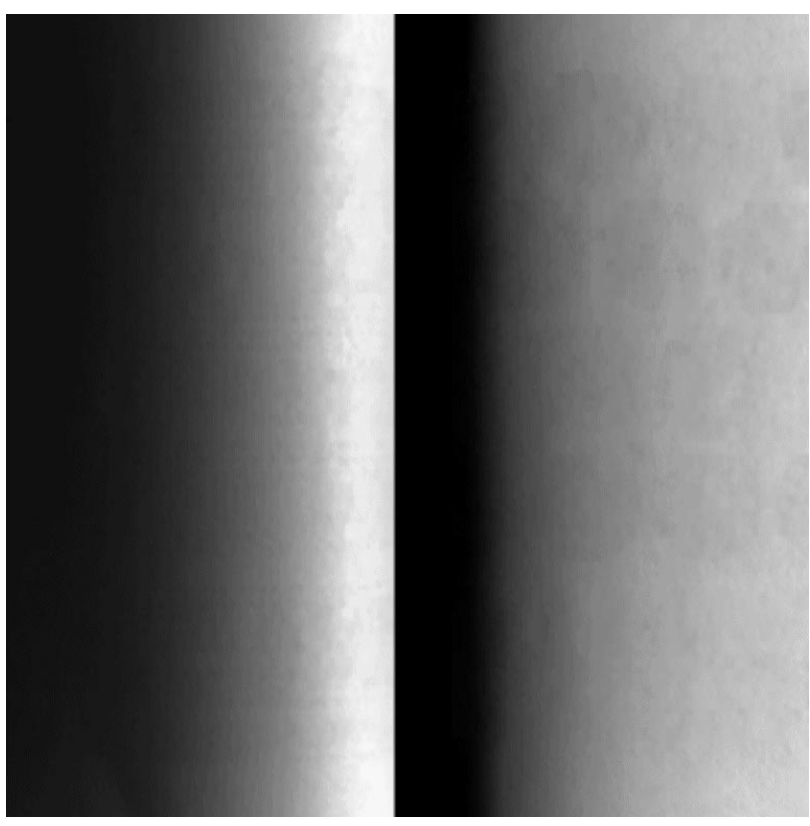

Figure 5. One of pure shade image extracted in preprocessing step.

preprocessing strategy is simple and ad hoc, acceptable results, being removed non-constant albedo and interreflections, have been obtained.

\subsection{Feed-back shape recovery}

Shape information can be obtained using theoretical recovery process for ideal case. However, in treating real images of book, more comprehensive algorithm is required because of existence of self-shadows, difficulty in determining occluding boundaries, and discontinuity of shade in folded part.

A feed-back process which encapsulates theoretical recovery process explained in Sect. 3.6 has been proposed for reliable shape recovery of book surface. Figure 6 shows flow diagram of proposed process.

Initial estimation of depth is done using eq. (6) by assuming shadowless over whole image. Using recovered shape, zone over the line of incident light ray is determined as shadowless on following condition

$$
\left|p^{D}\right| \leq \frac{\sqrt{\delta_{x i}^{2}+\delta_{y i}^{2}}}{\left|\delta_{x i}\right|}+\theta_{\text {thres }}, \quad \theta_{\text {thres }}>0 .
$$

Within shadowless zone, normalization of observed reflectance using recovered shape is done. This is done separately with respect to each image for compensating the intensity difference of two light sources.

In next process, same way as initial calculation is done on shadowless zone, but $p_{i}^{S}$ of eq. (7) is calculated by one shadowless image when another is self-

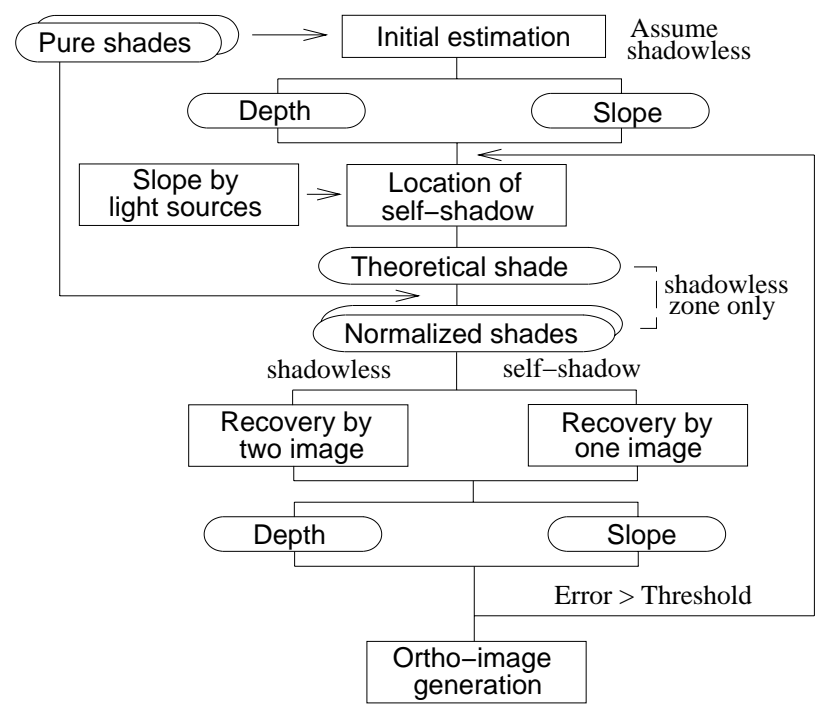

Figure 6. Feed-back process proposed for shape recovery of book surface.

shadowed. Combining depth from both zone, shadowed and non-shadowed, refined shape of book is recovered, and then this shape is used for next iteration.

This feed-back process is repeated until iteration error of depth becomes acceptably small.

During the shape recovery, column-wise means of slope and depth are calculated in order to avoid the bias by local noise. The recovery is proceeded from both margins to center of book considering discontinuity of shading of folded part. In folded part of book, where no direct light reaches, extrapolation of depth from known part is done.

\subsection{Generation of ortho-images}

Albedo images are generated by inverting eq. (4) and by using recovered shape with respect to both shade images. By combining two albedo images, pure albedo image of book surface is generated.

Finally, ortho-images of albedo and shape are generated by applying eq. (11) and by using recovered apparent shape.

\section{Simulations and Experiment}

\subsection{Simulations}

Shade images used for simulation of recovery have been generated on following condition.

- Shape : double cylinder

- Size : $512 \times 512$ with 45 pixels of maximum depth

- Distance to light sources : $( \pm 9000,0,3000)$ pixels

- Distance to camera : 4000 pixels

- Rate of interreflections : $1.0 \%$ 
Table 1. Results of simulation.

\begin{tabular}{|c|c|c|c|c|c|}
\hline Case & $\begin{array}{c}\text { Light } \\
\text { condition }\end{array}$ & $\begin{array}{c}\text { Observing } \\
\text { condition }\end{array}$ & $\begin{array}{c}\text { Shadow } \\
\text { condition }\end{array}$ & $\begin{array}{c}\text { Inter- } \\
\text { reflection }\end{array}$ & $\begin{array}{c}\text { Error } \\
(\%)\end{array}$ \\
\hline 1 & No & No & No & No & 79.6 \\
\hline 2 & Yes & Yes & No & No & 75.7 \\
\hline 3 & Yes & Yes & Yes & No & 41.7 \\
\hline 4 & Yes & Yes & Yes & Yes & 12.7 \\
\hline \multicolumn{7}{|c|}{ Error $=100 \times \frac{\left|Z_{\text {real }}-Z_{\text {recovered }}\right|}{Z_{\text {real }}}}$.
\end{tabular}

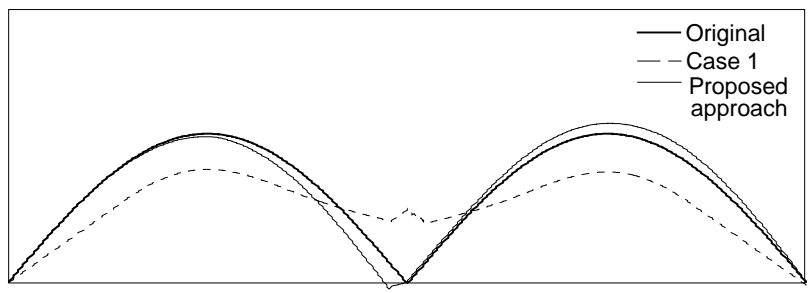

Figure 7. Simulation results of shape recovery.

- Condition : Perspective shading and observation, self-shadows, and interreflections

Table 1 and Fig. 7 show results of recovery. In each field of table, Yes means that correct condition has been considered, and No means ignoring the condition in recovery process. From the results of simulation, it can be expected that the proposed approach (case 4) will give smaller error in real experiments as well than conventional approaches which usually ignore perspective effects and self-shadows.

Another type of shade images assuming no effect of interreflections have been generated as comparison. The recovered result obtained by considering correct condition gives $3.5 \%$ of depth error. This error has been caused by the extrapolation error and the deformation of observed shape, that is $\frac{\partial Z}{\partial Y} \neq 0$, during perspective observation.

Therefore, error of case 4 can be interpreted as mainly coming from errors in removal of interreflections and successional error of extrapolation.

\subsection{Real experiment}

Figure 4 has been observed on below condition.

- Pixel size : $0.24 \mathrm{~mm}$

- Size of image : $998 \times 998$ pixels

- Distance to light sources : $( \pm 4504,0,1993)$ pixels

- Distance to camera : 3428 pixels

Shape recovery has been done using generated pure shade images (Fig. 5 and it's pair) by applying the proposed feed-back process. Convergence has been

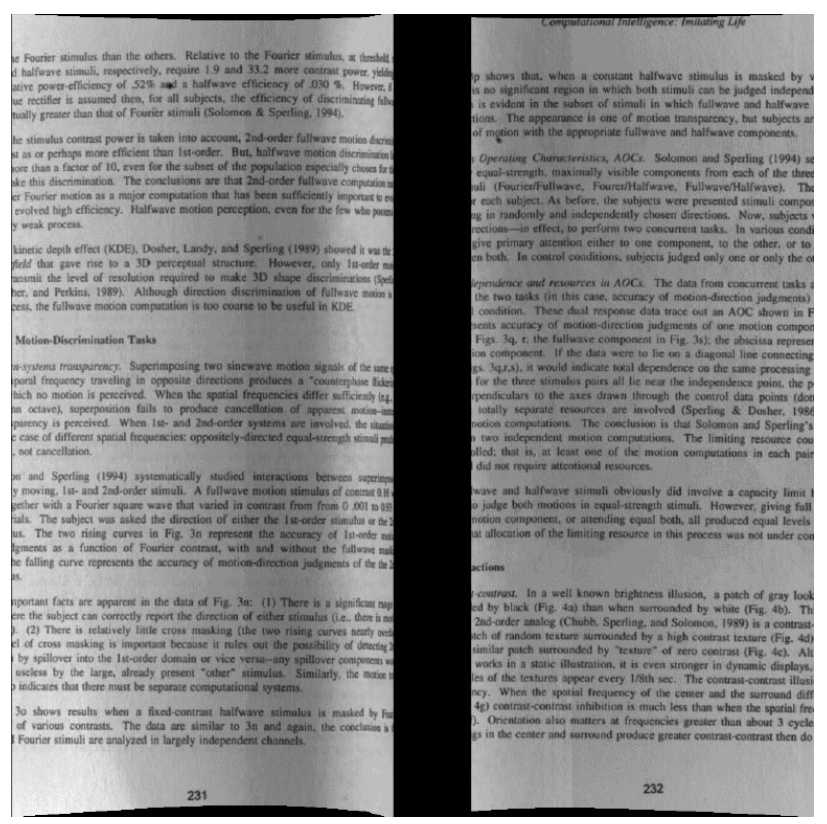

Figure 8. Finally obtained ortho-image.

reached at second iteration with respect to 0.1 pixel of iteration error and at third iteration with respect to 0.01 pixel of error. However, there has been no significant difference of recovered shape between the two.

Albedo of book has been calculated by inverting eq. (4) and using recovered shape. Recovered shape has been used for generating ortho-image of albedo as is shown in Fig. 8 and that of depth using eq. (11). Three dimensional perspective view of Fig. 9 has been generated in order to show recovered depth and albedo simultaneously.

Accuracy of shape recovery can be estimated from ortho-image of albedo indirectly by two ways.

One way is to check how much apparent warps caused by perspective observation have been corrected. The resulting image shows that much of apparent warps have been corrected acceptably over whole image although warps near folded part remains uncorrected.

Another way is to check how homogeneous the albedo of paper part is. Two albedo images can be obtained from two shade images. Two results are hard to be similar because reflectance of self-shadow zone is not complete black and because surface of book is not an ideal Lambertian object. Therefore, better result has been selected manually by comparing both results. By this problem, sudden variation of albedo can be observed in Fig. 8. The brightness information of fully shadowed parts where no direct light is reached has been removed as black in preprocessing step already by removing the effect of interreflections. As a result, 


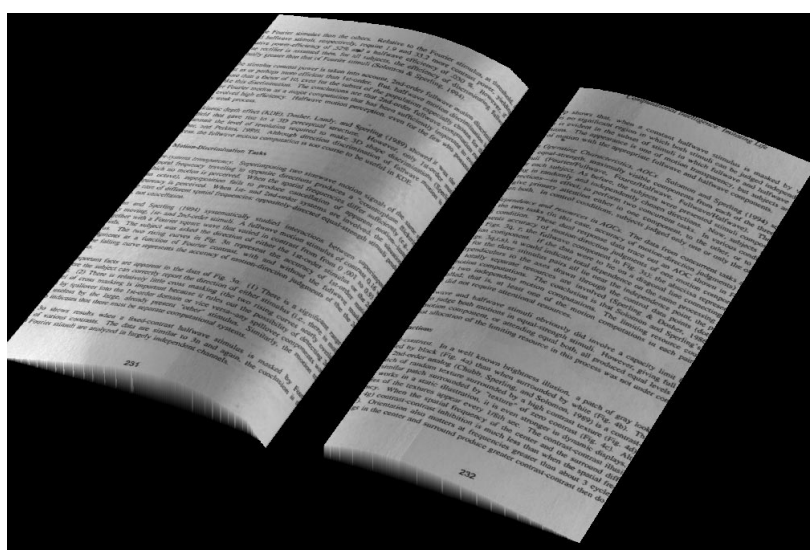

Figure 9. Three dimensional perspective view of book simulated from recovered albedo and depth.

central part of book remained black in Fig. 8 as well.

Despite of remained problems, it can be said that acceptable results have been obtained by considering difficulties of treated problem and by comparing to current technical status of shape from shading.

\section{Conclusions}

A theory and algorithms in recovering shape of book surface from shading on fully perspective condition have been discussed. The fundamental idea has been based on a divide-and-conquer strategy which divides complicated SFS problem into simplified ones and resolves implicit problem into explicit ones.

New concepts or approaches discussed in this paper are as follows.

- Division of problem into simplified ones

- Solution of fully perspective SFS problem

- Phenomenological model of interreflections

- Extraction of pure shade images

- Feed-back shape recovery overcoming self-shadow

The following self-estimation has been done.

First, resolving implicitness of problem is proper way in solving fully perspective SFS problem. Second, derived equations can be used as subset of other SFS approaches because of compatibility with orthographic condition. Third, proposed approach can not be used when there are severe noises during observation because the recurrence relation propagates recovered shape information. Finally, the proposed way of shape recovery is efficient in time because no iteration is required when there is no self-shadow.

It is still early to discuss about the accuracy of shape recovery using the results of simulations and real experiment. However, it may be said that the result of real experiment is acceptable because the main purpose of SFS is in fast recovery of shape.

The following further research can be proposed.

First, the surface of book is not ideal Lambertian object although it may be more closer than other objects. A way of correcting deviation by studying physical reflectance properties of a sheaf of bound paper can be proposed for further research. Second, the proposed approach can't be applicable for shape recovery of general object. Another invariance or constraint should be found for generalization of proposed approach. Third, the preprocessing algorithms are insufficient until now as has been shown by simulations and real experiments. Further researches shall be done in order to compensate these problems.

The proposed algorithms are expected to be useful in constructing the virtual library of rare books where the restriction of non-contact input is highly required.

\section{References}

[1] C. Cho and H. Minamitani. Obtaining 3-d shape from silhouette informations interpolated by photometric stereo. MVA, pages 147-150, 1994.

[2] B. Horn and R. Sjoberg. Calculating the reflectance map. Applied Optics, 18(11):1770-1779, 1979.

[3] K. Kanatani. Computational projective geometry. CVGIP (IU), 54(3):333-348, 1991.

[4] K. Kanatani and T. Chou. Shape from texture : General principle. Artificial Intelligence, 38:1-48, 1989.

[5] B. Kim and P. Burger. Depth and shape from shading using the photometric stereo method. CVGIP (IU), 54(3):416-427, 1991.

[6] K. Lee and C.-C. J. Kuo. Shape from shading with perspective projection. $C V G I P(I U), 59(2): 202-212$, 1994.

[7] W. Macchi and N. Lobo. Interreflections with rough surfaces. ICPR, pages 602-605, 1994.

[8] S. Nayer, K. Ikeuchi, and T. Kanade. Shape from interreflection. IJCV , 6(3):75-104, 1991.

[9] H. Ukida, T. Wada, and T. Matsuyama. 3d shape reconstruction of unfolded book surface from a scanner image. $M V A$, pages 409-412, 1994.

[10] T. Wada, H. Ukida, and T. Matsuyama. Recovering 3d shape of unfolded book surface from a scanner image (ii). Trans. IEICE, J78-D-II(2):311-320, 1995. Japanese.

[11] L. Wolff and E. Angelopoulou. Three-dimensional stereo by photometric ratio. JOSA (A), 11(11):30693078, 1994. 